der schwingenden Saite liegen die Verhältnisse so einfach, daß man ihrer mathematisch Herr werden konnte; aber schon in der Kristallographie ist die Sache genau so rätselhaft, wie im Planetensystem. Immerhin scheint aber hier ein Fall vorzuliegen, wo die Untersuchung des Mikrokosmos auch das Verständnis des Makrokosmos gefördert hat.

Innsbruck, t9 I I Mai.

Adalbert Prey.

Beobachtungen des Halleyschen Kometen 1909 c am Kiewer Refraktor.

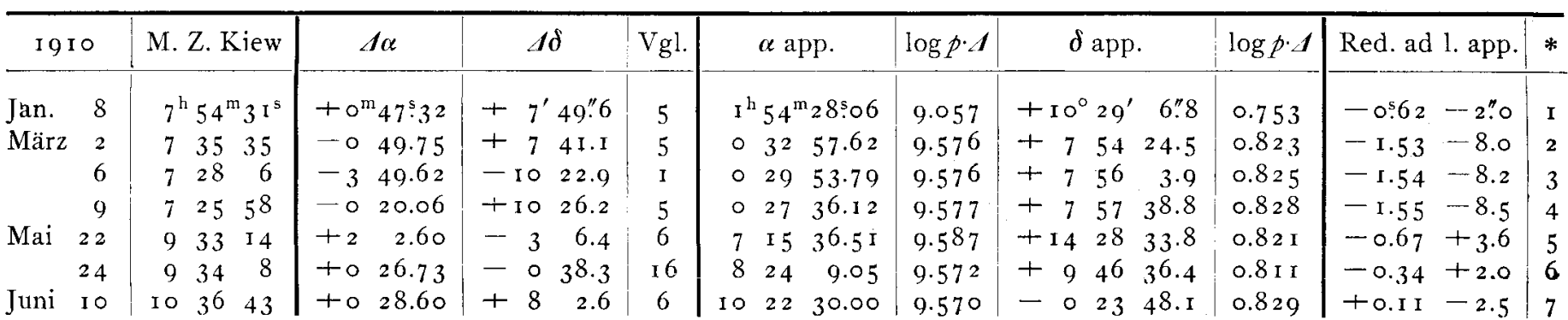

Mittlere Örter der Vergleichsterne.

\begin{tabular}{|c|c|c|c|}
\hline$*$ & $\alpha \quad 1910.0$ & $\delta 1910.0$ & Autorität \\
\hline I & $I^{h} 53^{m} 4 I^{s} \cdot 3^{6}$ & $+10^{\circ} 21^{\prime} 19^{\prime \prime} 2$ & $\mathrm{AGLp} \angle \mathrm{I} \quad 589$ \\
\hline 2 & $\circ 3.348 .90$ & +74651.4 & AG Lpz II I 99 \\
\hline 3 & ० 3344.95 & +8635.0 & 198 \\
\hline 4 & $\circ \quad 2757.73$ & $+7472 \mathrm{I} . \mathrm{I}$ & I $6 \mathrm{I}$ \\
\hline
\end{tabular}

Kreuzstabmikrometer. Antrittsmomente chronographisch registriert bei völlig dunklem Beobachtungsraume. Einfluß der Refraktion und Eigenbewegung beriicksichtigt.

Januar 8. Kälte $-20^{\circ}$ R. -- März 2. Komet sehr niedrig. - März I I. Komet auf dem von der Abenddämmerung stark beleuchteten Grunde äußerst schwach. Beobachtung unmöglich. - April 27 , morgens um $15^{\mathrm{h}} 45^{\mathrm{m}}$. Komet mit freien Auge sehr gut sichtbar. Im Sucher ist der »Kopf « parabolisch begrenzt, der Kern ist ungefähr im Brennpunkte dieser Parabel. Wegen heller Morgendämmerung von Vergleichsternen keine Spur, wie auch an allen folgenden Tagen. - Mai I 8 tm I $5^{\mathrm{h}}$ zahlreiche Wolken, gegen $17^{\mathrm{h}}$ blieben nur Wolken im Osten. Von $17^{\mathrm{h}} I^{\mathrm{m}}$ bis $18^{\mathrm{h}} 40^{\mathrm{m}}$ wegen angekündeter Möglichkeit eines Durchganges des Ko-

Kiew, Sternwarte, I9 I I Juni 20.

\begin{tabular}{|c|c|c|c|}
\hline$*$ & $a$ I9 10.0 & $\delta$ I 9 I0.0 & Autorität \\
\hline 5 & $7^{\mathrm{h}}$ I $3^{\mathrm{m}} 34^{\mathrm{s}} \cdot 5^{8}$ & $+14^{\circ} 31^{\prime} 36^{\prime \prime} 6$ & AG Lpz I $28 z 8$ \\
\hline 6 & $\begin{array}{lll}8 & 23 & 42.66\end{array}$ & +947 I 2.7 & AG Lpz II 4606 \\
\hline 7 & IO $22 \quad 1.29$ & $-\circ 3148.2$ & AG Nic 3060 \\
\hline
\end{tabular}

meten untersuche ich aufs sorgfältigste die Sonnenscheibe mit dem Refraktor (Vergr. erst I 45, dann 2 24 ). Eine Gruppe Sonnenflecken südlich vom Zentrum. Am südöstlichen Rande eine helle Fackel und am südwestlichen ein kleiner länglicher Fleck; vom Kometenkerne keine Spur. - Mai 24. Bei den letzten Vergleichungen bemerkte ich, daß der Komet scheinbar auf den Stern AG Lpz II 46 I 5 loszieht und ihn vielleicht bedecken wird, aber vorüberziehende Wölkchen vereitelten diese Beobachtung, denn als $u m$ I $0^{h} 3^{\mathrm{m}}$ I $6^{\mathrm{s}}$ der Komet wieder zum Vorschein kam, war er schon jenseits des Sterns und zwischen dem Sterne und dem Kometenkern ein kleiner Zwischenraum von ungefähr $\mathbf{I}^{\prime \prime}-\mathbf{2}^{\prime \prime}$, so daß nicht zu entscheiden war, ob er ihn genau zentral bedeckt hatte oder nur sehr nahe vorbeizog.

\title{
Observations of minor Planets and Comets
}

made at the Vassar College Observatory with the I 2 -inch refractor by Caroline E. Furness.

\begin{tabular}{|c|c|c|c|c|c|c|c|c|c|c|}
\hline I 909 & Greenw, M. T. & $\Delta \alpha$ & $\not \delta$ & Cp. & $\alpha$ app. & $\log p \cdot A$ & $\delta$ app. & $|\log p \cdot d|$ & Red.ad l. app. & $*$ \\
\hline
\end{tabular}

\begin{tabular}{|c|c|c|c|c|c|}
\hline \multirow[t]{2}{*}{ Oct. } & 25 & $\mathrm{I} 4^{\mathrm{h}} 45^{\mathrm{m}}$ I $2^{\mathrm{s}}$ & $-o^{m} 5 I^{s} \cdot 63$ & + & $0^{\prime} \quad 8 \cdot 3$ \\
\hline & 2 & I $3 \quad 16 \quad 22$ & to $4.67^{*}$ & - & 323.0 \\
\hline \multirow[t]{3}{*}{ Nov. } & I & I $53 I \quad 46$ & to $25.8 \mathrm{I} *$ & - & $\begin{array}{ll}3 & 10.5\end{array}$ \\
\hline & 5 & I $430 \quad 46$ & to $48.5^{2}$ & - & 27.6 \\
\hline & & I 530 & -o I $9.08 *$ & + & $\begin{array}{ll}5 & 2 \times .7\end{array}$ \\
\hline
\end{tabular}

(79) Eurynome.

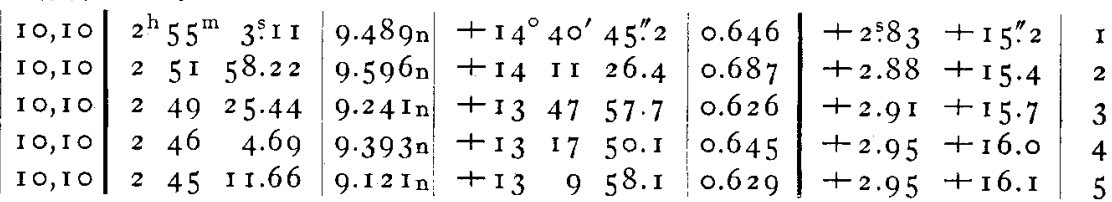

(8) Flora.

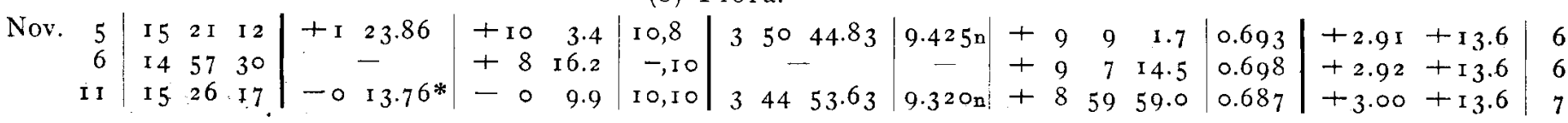




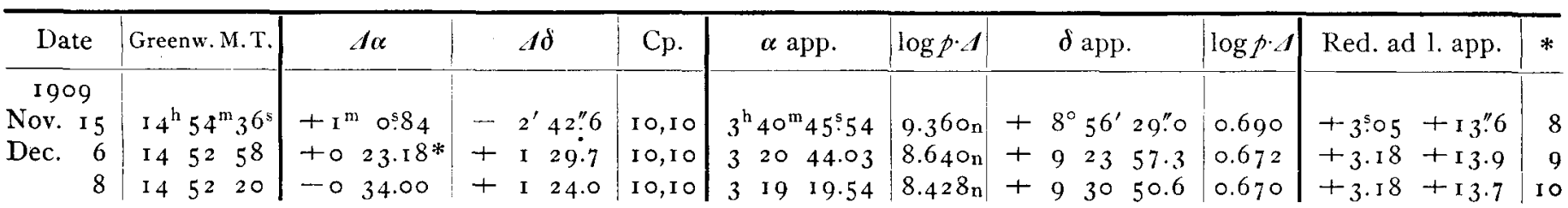

I9 10

Comet Halley r $909 \mathrm{c}$.

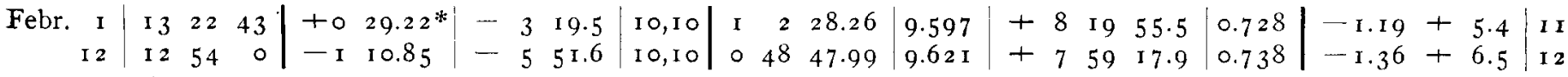

- $\Delta \alpha$ measured directly.

Mean places of the comparison stars.

\begin{tabular}{|c|c|c|c|}
\hline$*$ & $\alpha$ I 909.0 & $\delta$ I 909.0 & Authority \\
\hline I & $2^{\mathrm{h}} 55^{\mathrm{m}} 5 \mathrm{I} \cdot 9 \mathrm{I}$ & $+14^{\circ} 40^{\prime} 21^{\prime \prime} .7$ & AG Lpz I 896 \\
\hline 2 & 25 I 50.67 & +141434.0 & 876 \\
\hline 3 & $\begin{array}{lll}2 & 48 & 5^{6.72}\end{array}$ & $+13.505^{2.5}$ & 857 \\
\hline 4 & $245 \quad 13.22$ & +1320 & 830 \\
\hline 5 & $\begin{array}{lll}2 & 45 & 27.79\end{array}$ & $4 \quad 20.3$ & 832 \\
\hline 6 & $349 \quad 18.06$ & $+85^{8} 44.7$ & $\mathrm{AG} \mathrm{Lpz} \mathrm{II} \mathrm{I} 43^{6}$ \\
\hline 7 & $\begin{array}{lll}3 & 45 & 4.39\end{array}$ & +85955.3 & I 409 \\
\hline
\end{tabular}

\begin{tabular}{|c|c|c|c|}
\hline$*$ & $\alpha$ & $\delta$ & Authority \\
\hline 8 & $\begin{array}{c}1909.0 \\
3^{\mathrm{h}} 39^{\mathrm{m}} 4 \mathrm{I}^{\mathrm{s}} .65\end{array}$ & $\begin{array}{c}\text { 1 } 909.0 \\
+\quad 8^{\circ} 58^{\prime} 58.0\end{array}$ & AG Lpz II I 374 \\
\hline 9 & 32017.67 & +92213.7 & I 262 \\
\hline IO & $\begin{array}{l}31950.36 \\
1910.0\end{array}$ & $\begin{array}{c}+92912.9 \\
1910.0\end{array}$ & I 256 \\
\hline I I & $1 \quad 2 \quad 0.23$ & +8239.6 & AG Lpz II 401 \\
\hline 2 & $05^{\circ}$ & +8 & 308 \\
\hline
\end{tabular}

Comparison of observed places with ephemerides $(\mathrm{O}-\mathrm{C})$.

\begin{tabular}{|c|c|c|c|c|c|}
\hline 1909 & $A \alpha$ & $\langle\delta$ & 1909 & Ace & $\lambda \delta$ \\
\hline \multicolumn{3}{|c|}{$\begin{array}{l}\text { (79) Eurynome. } \\
\text { [Berl. Jahrb. I9 I ]. }\end{array}$} & Nov. $\begin{array}{r}5 \\
6\end{array}$ & $\begin{array}{l}-5.62 \\
-\quad 5.49\end{array}$ & $\begin{array}{l}-33^{\prime \prime} 6 \\
-33 \cdot \mathrm{I}\end{array}$ \\
\hline $\begin{array}{ll}\text { Oct. } & 25 \\
& 29\end{array}$ & $\begin{array}{l}-4.7 \mathrm{I} \\
-5.28\end{array}$ & $\begin{array}{l}-30.0 \\
-32.9\end{array}$ & \multicolumn{3}{|c|}{$\begin{array}{l}\text { (8) Flora. } \\
\text { thl. Not. 69.6 I } 9 \text { ]. }\end{array}$} \\
\hline Nov. I & -5.23 & -34.1 & Nov. 5 & - I 5.73 & $-I^{\prime} 7^{\prime \prime} \cdot 9$ \\
\hline
\end{tabular}

Poughkeepsie, N. Y., I 9 I I May 3 I.

\begin{tabular}{|c|c|c|}
\hline I 909 & $A \alpha$ & $\not \delta$ \\
\hline Nov. 6 & - & $-\mathbf{I}^{\prime} \quad 8.1 \mathrm{I}$ \\
\hline I I & $-\mathrm{r} 5^{\mathrm{s}} \cdot 77$ & $-I$ \\
\hline I 5 & - I 5.59 & $\begin{array}{ll}\text { - } & 6.7\end{array}$ \\
\hline $\begin{array}{ll}\text { Dec. } & 6 \\
8\end{array}$ & $\begin{array}{l}- \text { I } 4.3 \mathrm{I} \\
-\mathrm{I} 4.13\end{array}$ & 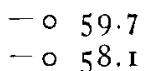 \\
\hline
\end{tabular}

Caroline E. Furness.

\section{Neue Bestätigung der Veränderlichkeit von RU (32) Cassiopeiae. \\ Vorläufige Mitteilung über einige neue veränderliche Sterne, sowie über $\varrho$ Cassiopeiae. \\ von P. Guthnick.}

RU Cassiopeiae. Die Veränderlichkeit dieses merkwürdigen Sternes wurde I904 von Miller Barr (A. J. 569) angezeigt und von Yendell (A. J. 572), sowie von Hartwig (V. J. S. I905) bald darauf bestätigt. Die Periode sollte sehr nahe 8 Stunden, die Amplitude $0^{\mathrm{m}} 4$ betragen und der Lichtwechsel von der Art desjenigen von U Pegasi sein. Später konnte trotz zahlreicher Bemühungen in Potsdam, CambridgeM., von H. Clemens, F. A. Parkhurst und Fordan nicht der geringste Helligkeitswechsel mehr konstatiert werden, so daß die Veränderlichkeit des Sterns wieder zweifelhaft wurde.

In diesem Jahre habe ich anläßlich einer größeren, versuchsweise schon vor einigen Jahren begonnenen, aber wieder unterbrochenen Beobachtungsreihe, über die später ausführlich in den A. N. berichtet werden soll, auch 32 Cassiopeiae wiederholt gemessen. Es hat sich herausgestellt, daß - der Lichtwechsel jetzt zweifellos wieder besteht und daß der Veränderliche in Wirklichkeit sehr wahrscheinlich vom Algoltypus ist, worauf auch das Spektrum (B 8) hinweist. Die Messungen lassen sich sehr befriedigend darstellen mit einer Periode, welche das dreifache der früher abgeleiteten ist, nämlich od9960. Als Epoche des Minimums ergibt sich mit ziemlicher Genauigkeit: I 9 I I Juli I 4.443 M. Z. Greenwich. Die Helligkeit im Minimum, bezogen auf den Vergleichstern 3 I Cassiopeiae $\left(5^{\mathrm{m}} \cdot 57\right)$, ist $6^{\mathrm{m}}$. I. Die normale Helligkeit habe ich noch nicht beobachten können, da gegenwärtig das Minimum sich hier über die ganze kurze Sommernacht erstreckt; sie ist aber jedenfalls nicht unter $5^{\mathrm{m}} \cdot 75$, die Amplitude wird daher wohl mindestens ${ }^{\mathrm{m}} \cdot 3$ betragen. Die Form der Lichtkurve ist die für gewöhnliche Algolsterne typische, d. h. symmetrisch und anscheinend ohne merkliche Dauer der Minimumhelligkeit. Die Dauer des ganzen Minimums beträgt mindestens 5 Stunden; außerhalb dieses Intervalles wird jedoch voraussichtlich die Helligkeitsänderung selbst für die schärfsten Messungen kaum mit Sicherheit feststellbar sein. Die nahe Übereinstimmung der Periodenlänge mit dem mittleren Sonnentag und die kurze Dauer der merklichen Helligkeitsschwankungen bringen es offenbar mit sich, daß die Minima für Orte auf der Erde, deren Längenunterschied nicht mehr als etwa 9 Stunden beträgt, lange Zeit hindurch gleichzeitig fast völlig in den Tag fallen und daher nicht bemerkt werden können. 